\title{
Multi-wavelength Characterization of Stellar Flares on Low-mass Stars using SDSS and 2MASS Time Domain Surveys
}

James R. A. Davenport

Western Washington University, james.davenport@wwu.edu

Andrew C. Becker

Adam F. Kowalski

Suzanne L. Hawley

Sarah J.Schmidt

See next page for additional authors

Follow this and additional works at: https://cedar.wwu.edu/physicsastronomy_facpubs

Part of the Stars, Interstellar Medium and the Galaxy Commons

\section{Recommended Citation}

Davenport, James R. A.; Becker, Andrew C.; Kowalski, Adam F.; Hawley, Suzanne L.; Schmidt, Sarah J.; Hilton, Eric J.; Sesar, Branimir; and Cutri, Roc, "Multi-wavelength Characterization of Stellar Flares on Low-mass Stars using SDSS and 2MASS Time Domain Surveys" (2012). Physics \& Astronomy. 20.

https://cedar.wwu.edu/physicsastronomy_facpubs/20 
Authors

James R. A. Davenport, Andrew C. Becker, Adam F. Kowalski, Suzanne L. Hawley, Sarah J. Schmidt, Eric J. Hilton, Branimir Sesar, and Roc Cutri 


\title{
MULTI-WAVELENGTH CHARACTERIZATION OF STELLAR FLARES ON LOW-MASS STARS USING SDSS AND 2MASS TIME-DOMAIN SURVEYS
}

\author{
James R. A. Davenport ${ }^{1}$, Andrew C. Becker ${ }^{1}$, Adam F. Kowalski ${ }^{1}$, Suzanne L. Hawley ${ }^{1}$, Sarah J. Schmidt ${ }^{1}$, \\ ERIC J. Hilton ${ }^{1,4}$, Branimir Sesar ${ }^{2}$, AND Roc CUtri ${ }^{3}$ \\ ${ }^{1}$ Department of Astronomy, University of Washington, Box 351580, Seattle, WA 98195, USA; jrad@ astro.washington.edu \\ ${ }^{2}$ Division of Physics, Mathematics and Astronomy, Caltech, Pasadena, CA 91125, USA \\ ${ }^{3}$ Infrared Processing and Analysis Center, California Institute of Technology, Pasadena, CA 91125, USA \\ Received 2011 September 17; accepted 2012 January 10; published 2012 March 6
}

\begin{abstract}
We present the first rates of flares from $M$ dwarf stars in both red optical and near-infrared (NIR) filters. We have studied $\sim 50,000 \mathrm{M}$ dwarfs from the Sloan Digital Sky Survey (SDSS) Stripe 82 area and $1321 \mathrm{M}$ dwarfs from the Two Micron All Sky Survey (2MASS) Calibration Scan Point Source Working Database that overlap SDSS imaging fields. We assign photometric spectral types from M0 to M6 using $(r-i)$ and $(i-z)$ colors for every star in our sample. Stripe 82 stars each have 50-100 epochs of data, while 2MASS Calibration stars have 1900 epochs. From these data we estimate the observed rates and theoretical detection thresholds for flares in eight photometric bands as a function of spectral type. Optical flare rates are found to be in agreement with previous studies, while the frequency per hour of NIR flare detections is found to be more than two orders of magnitude lower. An excess of small-amplitude flux increases in all bands exhibits a power-law distribution, which we interpret as the result of flares below our detection thresholds. In order to investigate the recovery efficiency for flares in each filter, we extend a two-component flare model into the NIR. Quiescent M0-M6 spectral templates were used with the model to predict the photometric response of flares from $u$ to $K_{s}$. We determine that red optical filters are sensitive to flares with $u$-band amplitudes $\gtrsim 2 \mathrm{mag}$, and NIR filters to flares with $\Delta u \gtrsim 4.5$ mag. Our model predicts that M0 stars have the best color contrast for $J$-band detections, but M4-M6 stars should yield the highest rate of NIR flares with amplitudes of $\Delta J \geqslant 0.01 \mathrm{mag}$. Characterizing flare rates and photometric variations at longer wavelengths is important for predicting the signatures of $\mathrm{M}$ dwarf variability in next-generation surveys, and we discuss their impact on surveys such as the Large Synoptic Survey Telescope.
\end{abstract}

Key words: stars: flare - stars: low-mass

Online-only material: color figures

\section{INTRODUCTION}

The study of flares on $\mathrm{M}$ dwarfs has a rich history, spanning more than seven decades (e.g., van Maanen 1940; Joy \& Humason 1949). The similarity of these events to resolved flares on the Sun was recognized many years ago (e.g., Lovell 1969). The seminal paper by Kunkel (1970) laid the groundwork for our present understanding of the broad multi-wavelength properties of M dwarf flares. The basic two-component flare spectral model ( $\mathrm{H}$ recombination + warm blackbody continuum) presented in Kunkel (1970) is useful for estimating the total energy and stellar surface extent of flares in the optical regime (Hawley \& Pettersen 1991; Kowalski et al. 2010). However, this model does not specifically address the detailed spectral features observed during flares, such as line emission from $\mathrm{H}, \mathrm{He}$, He II, and Ca II H\&K, nor the evolution of the spectral energy distribution (SED) as the flare cools and decays (Fuhrmeister et al. 2008). A partnership of multi-wavelength photometric and spectroscopic time-domain observing campaigns (e.g. Byrne 1989; Hawley et al. 2003) and detailed radiative hydrodynamical modeling (Allred et al. 2006) are required to extend our understanding of stellar flare emission physics.

West et al. (2008) found that the fraction of M dwarfs that show evidence of magnetic activity as defined by $\mathrm{H} \alpha$ emission decreases with height above the Galactic plane. This is thought

\footnotetext{
4 Current Address: Institute for Astronomy and Department of Geology and Geophysics, University of Hawaii, 2680 Woodlawn Drive, Honolulu, HI 96822, USA.
}

to be an age effect, with the older, less active stars lying further from the plane due to dynamical heating and radial migration within the disk (West et al. 2008; Loebman et al. 2011). Flares may play an important role in the main-sequence stellar evolution for low-mass stars, and correlating $\mathrm{M}$ dwarf flare rates with mass and metallicity promises to yield an effective constraint on the ages of stellar populations.

Models and observations of the initial mass function indicate that $\mathrm{M}$ dwarfs are the primary stellar component in the Galaxy by number (Bochanski et al. 2010). Recent large-scale surveys, such as the Sloan Digital Sky Survey (SDSS; York et al. 2000), have photometrically identified millions of M dwarfs (Bochanski et al. 2010). These stars make excellent tracers of nearby galactic structure due to their high number densities (Bochanski et al. 2007a), and future survey missions, like the Large Synoptic Survey Telescope (LSST; Ivezic et al. 2008), will detect M dwarfs to nearly the full extent of the Galaxy, using longer wavelength bandpasses and deep repeat imaging. LSST will map the sky approximately 1000 times over 10 years using ugrizy bands and is expected to detect flares both as variability in previously quiescent stars and as transient emission from previously undetected sources. This foreground "fog" of characteristically blue transient events will prove a significant contaminant to any statistical measurements of variability from cosmological sources (Rau et al. 2008).

Kowalski et al. (2009, hereafter K09) used the SDSS Stripe 82 time-domain photometric data (Sesar et al. 2007) to look for flares from $\sim 50,000 \mathrm{M}$ dwarf light curves in the $u$ and 
$g$ bands. From these sparse light curves they were able to recover a flare rate consistent with those found from dedicated photometric monitoring campaigns (e.g., Lacy et al. 1976). The rate of flares was found to decrease with peak flare luminosity and increase with stellar spectral type (redder colors). Hilton et al. (2010b) found serendipitous flares in the time-resolved SDSS spectroscopic data from $\sim 38,000 \mathrm{M}$ dwarfs across the entire SDSS Data Release 6 footprint (Adelman-McCarthy et al. 2008). They confirmed the increase of the flare duty cycle with later spectral type and further investigated the Galactic height dependence of flaring activity, finding that the stars that flared were even more closely confined to the Galactic plane than the stars with $\mathrm{H} \alpha$ activity.

The few studies of M dwarf flares in the near-infrared (NIR) that have been previously attempted have not found a consistent relationship to the optical emission. Rodonò \& Cutispoto (1988), for example, measured a marginal anti-correlation between optical $U$-band and infrared $K$-band photometry of two flares. Similarly, Panagi \& Andrews (1995) found no $K$-band enhancement during $26 \mathrm{M}$ dwarf flares that were observed simultaneously in the $U$ band. Observations of flares on the Sun in the NIR offer a more promising avenue. Xu et al. (2006) studied the luminosity of solar flares at $1.6 \mu \mathrm{m}$, the $\mathrm{H}^{-}$ opacity minimum, and found a correlation with hard X-ray and white-light optical emission for two X-class flares. Their data indicated that the NIR band reached peak luminosity after the $\mathrm{X}$-ray and was responding to secondary heating, possibly from chromospheric back-warming.

Modern $\mathrm{M}$ dwarf flare studies are expanding in both wavelength and temporal coverage to gain insight into stellar flare physics, as well as the contaminating impact these events have on detecting other sources of variability. Hawley et al. (2003) found a correlation between flare energies in the optical, nearUV, and X-ray. Schmidt (2012) has observed flaring M dwarfs with NIR spectroscopy to characterize the correlation between NIR emission lines, such as the H Paschen series, and blue optical broadband flux during flares. Tofflemire et al. (2012) have undertaken the most comprehensive simultaneous optical and NIR photometric observing campaign for flares to date to determine the effects of flares on exoplanet detection around $\mathrm{M}$ dwarfs. These high-cadence and high-precision data place upper limits on the broadband NIR emission from several medium $u$-band flares from M3 and M4 stars with $~ 3.9$ mmag photometric precision.

In this paper we seek to extend the analysis of K09 using the time-domain data from the Two Micron All Sky Survey (2MASS) Calibration Point Source Working Database (hereafter Cal-PSWDB), as well as the SDSS Stripe 82 data. The combination of these data allows us to statistically characterize for the first time the rate of broadband $\mathrm{M}$ dwarf flares in red optical and NIR wavelengths. Our data are outlined in Section 2. Variability statistics are discussed in Section 3. Flare rates as a function of both spectral type and observed passband are presented in Section 4. A spectroscopic model of an M dwarf flare is given in Section 5, and the predicted sensitivity to finding flares in each band in Section 6. Finally, a concluding discussion is given in Section 7.

\section{TIME-DOMAIN DATABASES}

Our data are drawn from the SDSS and 2MASS photometric time-domain databases. Every M dwarf in our study has singleepoch SDSS Data Release 7 (DR7; Abazajian et al. 2009) photometry, used to determine photometric spectral types and

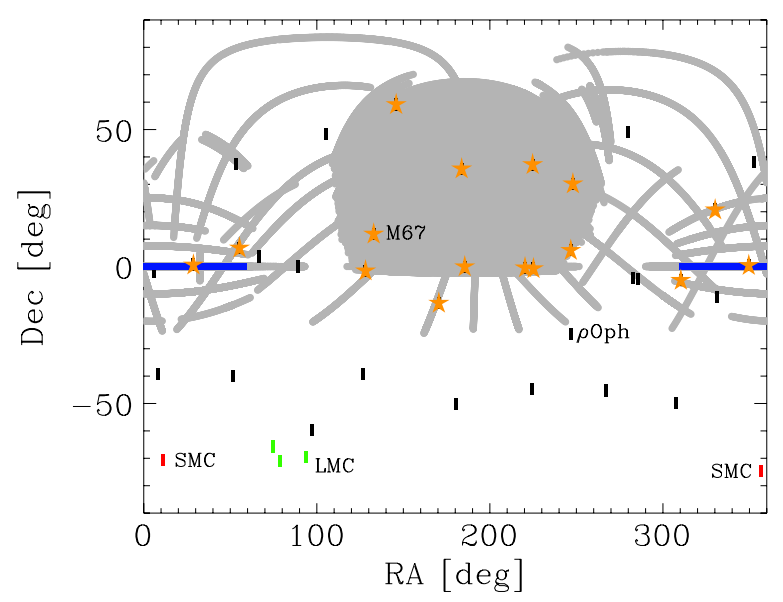

Figure 1. Distribution of the Cal-PSWDB tiles across the sky, with a few notable fields labeled. LMC fields are shown in green, SMC in red. Orange stars denote Cal-PSWDB tiles with matches to DR7. The SDSS footprint is shown for reference in gray, with the Stripe 82 data used shown in blue.

(A color version of this figure is available in the online journal.)

distances. Here we describe the selection criteria and spatial matching of our time-domain samples.

\subsection{MASS Cal-PSWDB}

Our near-infrared data come from the 2MASS Cal-PSWDB. Full details are given in the online Explanatory Supplement ${ }^{5}$ and Cutri et al. (2006). In summary, the repeat imaging was obtained to provide consistent photometric calibration for 2MASS over the four years of its operation. There were 35 regular calibration tiles spread across the sky, with each tile spanning approximately 8.5 in R.A. and $1^{\circ}$ in Decl. One of these 35 tiles was visited hourly during survey operations by the 2MASS telescopes, with simultaneous imaging in the $J, H, K_{s}$ bands. This sequence produced between 562 and 3692 epochs for these calibration fields. Five additional tiles were imaged in the LMC and SMC with fewer epochs. A total of 191 million point-source measurements are contained in the database. A pilot study with the Cal-PSWDB by Becker et al. (2008) revealed a 2.6 day lowmass eclipsing binary system. Plavchan et al. (2008b) used the Cal-PSWDB data to search for periodic variability of low-mass stars, finding several eclipsing binary candidates. Additionally, Plavchan et al. (2008a) found a periodic signature in CalPSWDB from a possible triple young stellar object in the $\rho$ Ophiuchus star-forming region.

We entered the Cal-PSWDB point-source measurements into a MySQL database to allow rapid matching of these data to other data sets. The 191 million measurements were clustered into individual objects using the spatial clustering algorithm, OPTICS (Ankerst et al. 1999), creating light curves for 113,030 unique objects. Of the 35 primary Cal-PSWDB tiles, 16 were coincident with the SDSS Data Release 7 photometric catalog (hereafter DR7; Abazajian et al. 2009), and we found 11,445 matched point-source objects using a 2 ". 5 radius. Figure 1 shows the positions of the 40 Cal-PSWDB tiles as compared to the SDSS photometric footprint. An optical-NIR $\left(g-K_{s}, i-J\right)$ color-color diagram for the matched point-source objects is shown in Figure 2, with 1963 galaxies and 9369 stars. The SDSS OBJ_TYPE flag was used to distinguish between stars and galaxies. We removed the 113 objects with unknown object

\footnotetext{
5 http://www.ipac.caltech.edu/2mass/releases/allsky/doc/seca4_1.html
} 
Table 1

Outline of Sample Selection Cuts for the Cal-PSWDB Data

\begin{tabular}{lccc}
\hline \hline Step & Number & Cut(s) & Description \\
\hline 1. & 11,445 (objects) & Cal-PSWDB - DR7 Match \\
2. & $5.75 \times 10^{6}$ (epochs) 4731 (stars) & $0 \leqslant$ SpType $\leqslant 6$, OBJ_TYPE $=6$ & M dwarfs only \\
3. & $2.51 \times 10^{6}$ (epochs) 1321 (stars) & PH_QUAL $=$ A, & High-S/N Epochs \\
& & $\sigma_{\text {RA, Dec }<0^{\prime \prime} 25}$ & Deblending \\
4. & $1.14 \times 10^{6}(J H$ epochs $)$ & $J \leqslant 17, H \leqslant 16.5, \sigma_{J}<0.2, \sigma_{H}<0.25$ & Good Photometry \\
5. & $9.08 \times 10^{5}(H K$ epochs $)$ & $H \leqslant 16.5, K \leqslant 16, \sigma_{H}<0.25, \sigma_{K}<0.3$ & Good Photometry \\
\hline
\end{tabular}

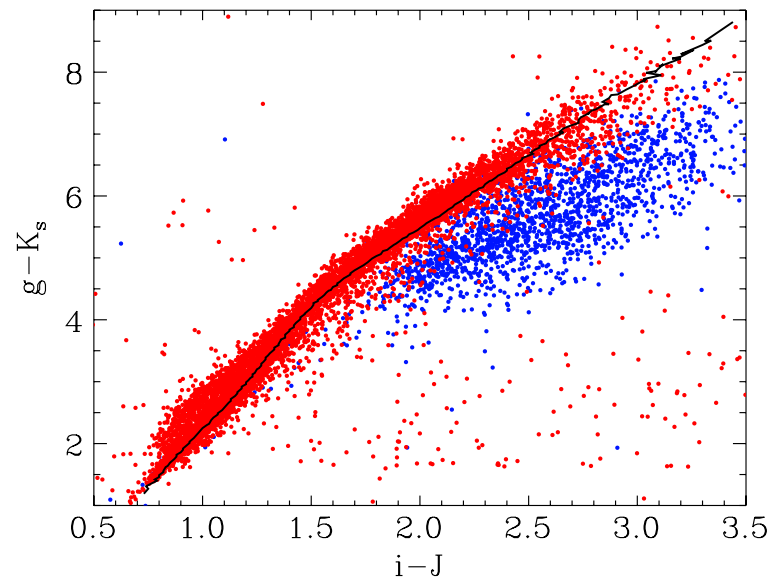

Figure 2. Color-color diagram for the 10,000 point-source objects with matches in both DR7 and Cal-PSWDB. Stars, as determined by the SDSS OBJ_TYPE flag, are shown as red circles, galaxies as blue. These two populations nicely separate. The Covey et al. (2007) fiducial stellar locus is shown for reference and follows closely the stellar objects in our sample.

(A color version of this figure is available in the online journal.)

type $\left(\mathrm{OBJ} \_\mathrm{TYPE}=0\right)$. The distribution of stars in our sample is well fit by the Covey et al. (2007) fiducial stellar locus.

We imposed magnitude limits of $r<23$ and $z<23$ and standard SDSS photometric flag cuts to ensure good SDSS DR7 photometry. M dwarfs were selected and spectral types assigned using $(r-i)$ and $(i-z)$ colors and the covariance matrix technique as outlined in K09. We found 4860 stars with photometric spectral types later than M0, with very few stars in the M7-M9 range due to their faint apparent magnitudes. The resulting 4731 M0-M6 stars had a total of $5.75 \times 10^{6}$ pointsource measurements in Cal-PSWDB. Only 12 of our stars were in the SDSS spectroscopic M dwarf catalog (West et al. 2011), so we rely solely on the SDSS photometry for classification.

Covey et al. (2008) determined color and magnitude criteria to cleanly separate giant and dwarf stars in SDSS and 2MASS data. Potential contamination by giants or stars with spectral types earlier than M0 was very small, with only 65 stars (less than 5\%) from our Cal-PSWDB sample falling outside the cuts $(i-J>1.25, J>12$ ) outlined by Covey et al. (2008). Our targets that fail these cuts are predicted to have less than a $\sim 10 \%$ chance of being giants, according to Covey et al. (2008).

Photometric calibration of the Cal-PSWDB was not handled in the same manner as the main 2MASS, and as such many caveats for this database exist. Issues with source blending and photometric zero-point errors are present as described in Appendix 4 of the 2MASS Explanatory Supplement. To minimize these effects, we restricted our analysis to epochs with the Cal-PSWDB flag PH_QUAL = A, which required every $J H K_{s}$ measurement to have a signal-to-noise ratio $(\mathrm{S} / \mathrm{N})$ of at least 10 .
To ensure that each DR7 source matched to only a single Cal-PSWDB object, we conservatively removed all objects with a spatial standard deviation in their Cal-PSWDB data of $\sigma_{s}=\sqrt{\sigma_{\mathrm{RA}}^{2}+\sigma_{\mathrm{Dec}}^{2}} \geqslant 0^{\prime \prime} .25$. This cut limited the effects of source confusion and deblending but also removed any high proper motion objects from our sample. We additionally excluded epochs where photometric errors exceeded $\sigma_{J} \geqslant 0.2, \sigma_{H} \geqslant$ 0.25 , or $\sigma_{K} \geqslant 0.3$. Our final Cal-PSWDB sample contained 1321 M dwarfs with $\sim 2 \times 10^{6}$ epochs, for an average of $\sim 1900$ good epochs per star. The selection of our Cal-PSWDB sample is detailed in Table 1.

\subsection{SDSS Stripe 82}

In addition to the matched time-domain $J H K_{s}$ data from Cal-PSWDB, we analyzed M dwarfs from the SDSS Stripe 82 repeat scan database (Sesar et al. 2007) to obtain time domain data in the ugriz bands (Fukugita et al. 1996). These data were previously used by K09 to search for flares in the $u$ and $g$ bands, and we adopt their sample as described in their Table 2. Briefly, these data contain 50,130 photometrically selected M dwarfs, with 1.9 million total photometric measurements. The stars were required to have good $u$-band magnitudes $(u<22)$, which limited the sample to relatively bright M dwarfs. Only 429 of these objects have matches in the Cal-PSWDB database, due to the limited spatial overlap. We measure the flare rate independently in each filter to build statistics using the entire population from each time-domain survey.

The spatial location of the SDSS equatorial Stripe 82 data is shown in Figure 1 in blue. Figure 3 gives an example of the eightband light curves we have produced for a representative M0 star with both Stripe 82 and Cal-PSWDB photometry, illustrating the differences in time coverage and cadence between the two surveys. This star had 2922 epochs of data in each of the $J H K_{s}$ bands and 70 epochs in each of ugriz. The time units for all of our SDSS and 2MASS data have been converted to barycentric dynamical time $\left(\mathrm{BJD}_{\mathrm{TDB}}\right)$ and are presented as a modified barycentric Julian date (BJD-2400000.5). Figure 4 shows the numbers of stars in each spectral-type bin for our Stripe 82 and Cal-PSWDB samples.

\section{THE $\Phi$ STATISTIC}

Discerning true variability from background noise requires sophisticated statistical techniques. Following K09, we utilize a modified Welch-Stetson variability index, $\Phi$, to search for flares in our sample (Welch \& Stetson 1993; Stetson 1996). This statistic uses measurements in two bands to separate real stellar variability from the intrinsic scatter in a light curve. For the $n$th measurement of a light curve in $J$ and $H$ bands, for example, $\Phi$ is defined as

$$
\Phi_{J H}(n)=\left[\frac{m_{J}(n)-\left\langle m_{J}\right\rangle}{\sigma_{J}(n)}\right]\left[\frac{m_{H}(n)-\left\langle m_{H}\right\rangle}{\sigma_{H}(n)}\right],
$$




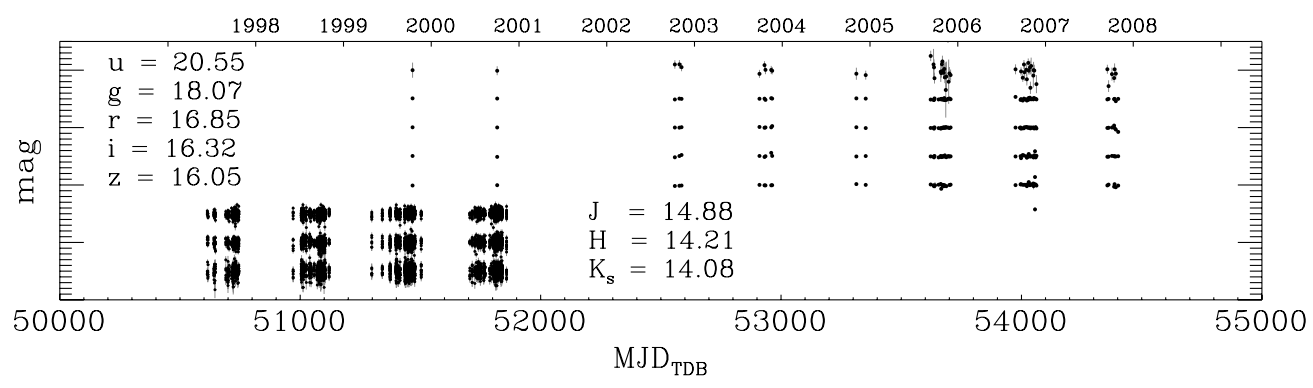

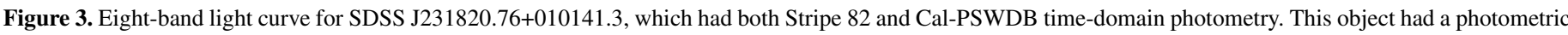

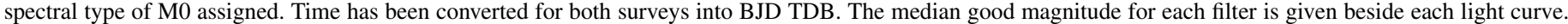
For reference the calendar year is given on the top-axis.

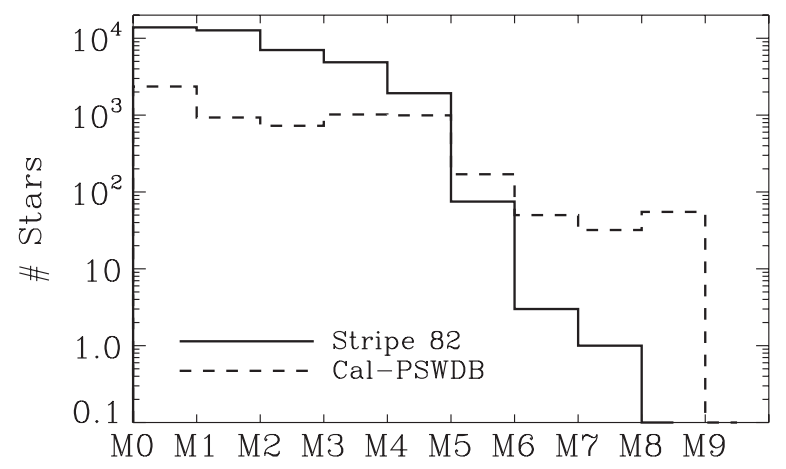

Figure 4. Distribution of spectral types for our Stripe 82 and Cal-PSWDB samples. Due to the lack of stars in the M7-M9 bins, we have only performed our analysis on M0 through M6 sub-types.

where $m(n)$ is the apparent magnitude at epoch $n,\langle m\rangle$ the median magnitude over all epochs, and $\sigma(n)$ the photometric error at epoch $n$. When the magnitude increases or decreases for both bands simultaneously, $\Phi$ yields a positive value. Conversely, when the magnitude from one band is higher than normal while the other is diminished, $\Phi$ is negative. For purely random noise between the two filters, this index would yield a symmetrical distribution about $\Phi=0$. Excesses of positive $\Phi$ values represent correlated variability, from events such as flares or eclipses.

We computed the $\Phi$ statistic for all pairs of bandpasses "adjacent" in wavelength space (e.g., $\left.\Phi_{u g}, \Phi_{J H}\right)$ for every epoch in our samples. Since the SDSS and 2MASS data were not obtained contemporaneously (see Figure 3), we do not compute $\Phi_{z J}$. Figure 5 shows the $\Phi$ histograms for all objects in our Stripe 82 and Cal-PSWDB samples. The black line indicates the $\Phi>0$ data, hereafter denoted $\Phi^{+}$, while the blue line shows the $|\Phi<0|$ data, hereafter $\Phi^{-}$.

As the sampling cadence of Stripe 82 and Cal-PSWDB surveys was much longer than typical flare timescales of minutes to hours (Moffett 1974), our flare search relies on single-epoch outliers in the light curves. In order to act as an effective variability discriminant the two bandpasses making up the $\Phi$ statistic must be imaged nearly simultaneously to capture the same physical event. The 2MASS $J H K_{s}$ bands were imaged simultaneously (Skrutskie et al. 2006), allowing the photometry to be directly compared for each $7.8 \mathrm{~s}$ exposure. The SDSS used a drift-scanning technique that produced separate $52.4 \mathrm{~s}$ exposures in each filter taken in the order $r, i, u, z, g$ (York et al. 2000). As a result, the $\Phi_{r i}$ values, for example, come from measurements that were separated by only $\sim 1$ minute, while the $\Phi_{g r}$ values are derived from data that were separated by almost 5 minutes. We searched for flares in each of the six $\Phi$ distributions separately but recognize the limitation that flares with timescales of only a few minutes may not have been imaged by all SDSS bands. As such, these rapid events would not yield large $\Phi$ values and may fall below our detection thresholds.

To remove large $\Phi$ values from the sample that resulted from a decrease of flux in both bands, for example due to photon loss from clouds, eclipses, or starspots, we required all flare candidate epochs in our investigation to have $m<\langle m\rangle$ for the bluer (shorter wavelength) filter in each of the $\Phi^{+}$and $\Phi^{-}$distributions. This removed approximately half the epochs in each distribution, as expected. As discussed in K09, the $\Phi^{-}$distribution, also referred to as the "null" distribution, is representative of uncorrelated noise in the light curve. The $\Phi^{-}$ distribution was still well modeled by Gaussian noise in all filters after the removal of half the epochs. Comparison of the $\Phi^{+}$distribution with the $\Phi^{-}$yields excesses in the $\Phi^{+}$due to candidate flaring epochs.

\section{OPTICAL AND NIR FLARE RATES}

In this section, we explore the selection of flares from our light curves and the luminosities of the flare candidate epochs.

\subsection{Observed Rates}

As discussed above, given our sparse sampling we assume that each flare is observed in only one epoch of our data. A threshold value for $\Phi^{+}$must be chosen from the null distributions to separate flare epochs from the quiescent epochs with statistical fluctuations. If too high a threshold is chosen, we may lose valuable weak signal in the redder bandpasses by disregarding real flare events. However, too low a threshold will add significant false positives to the resulting flare rates. Using the False Discovery Rate method (Miller et al. 2001) on the $\Phi_{u g}^{-}$distribution, K09 determined that a conservative cutoff for flares was $\Phi_{u g}^{+} \geqslant 100$. This ensured that less than $10 \%$ contamination from non-flaring epochs would be included in their final analysis. We performed the False Discovery Rate analysis to limit the contamination to $10 \%$ in all six of our $\Phi^{-}$ distributions and determined $\Phi$ thresholds of $(100,100,120$, $500,50,50)$ for $\left(\Phi_{u g}, \Phi_{g r}, \Phi_{r i}, \Phi_{i z}, \Phi_{J H}, \Phi_{H K_{s}}\right)$, respectively. The $\Phi^{-}$distributions using 2MASS filters in Figure 5 decline rapidly at $\Phi \sim 20$, nearly an order of magnitude lower than for the optical filters. This is indicative of the lower intrinsic stellar variability seen at these wavelengths and the larger photometric errors in 2MASS. The $\Phi$ thresholds are shown as dashed vertical lines in Figure 5. Only one candidate flaring epoch, less than $1 \%$ of our final sample, was found to occur on a star that did not pass the Covey et al. (2008) cuts for giants or earlier-type stars. 


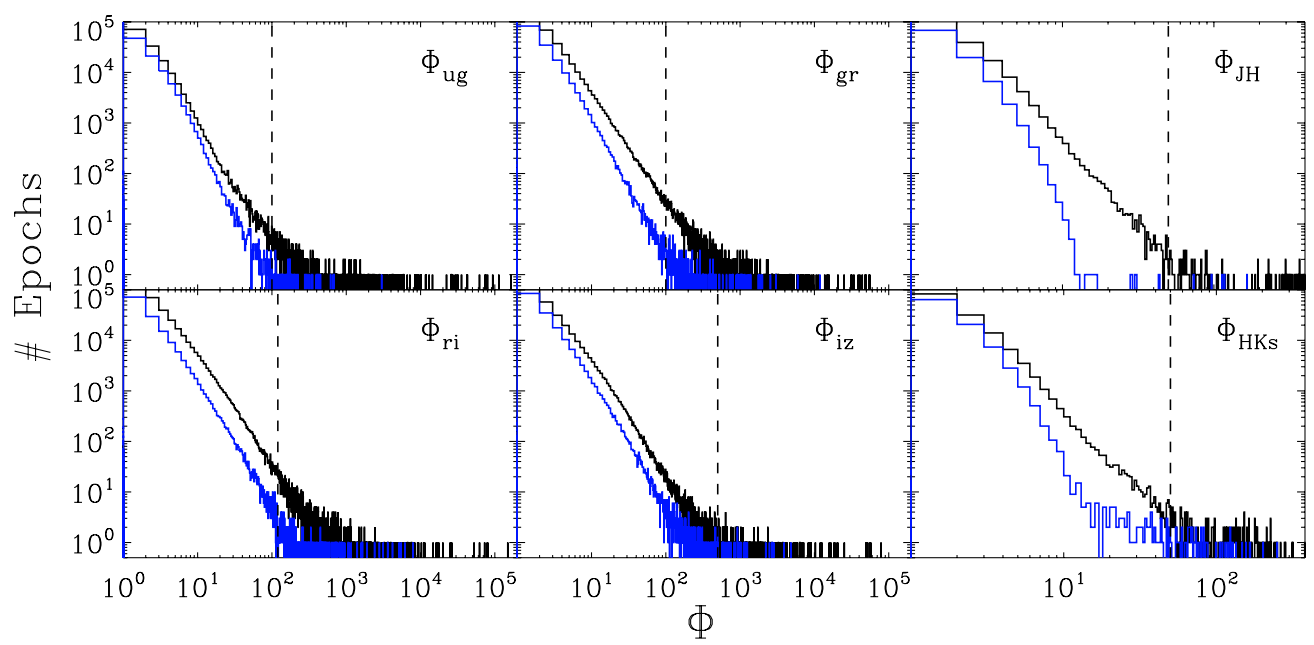

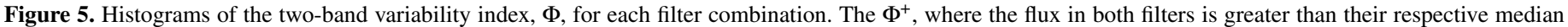

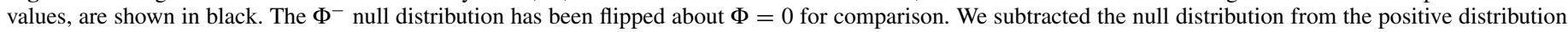

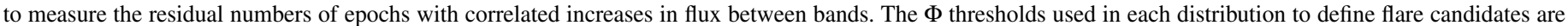
shown as vertical dashed lines. These are described in the text.

(A color version of this figure is available in the online journal.)

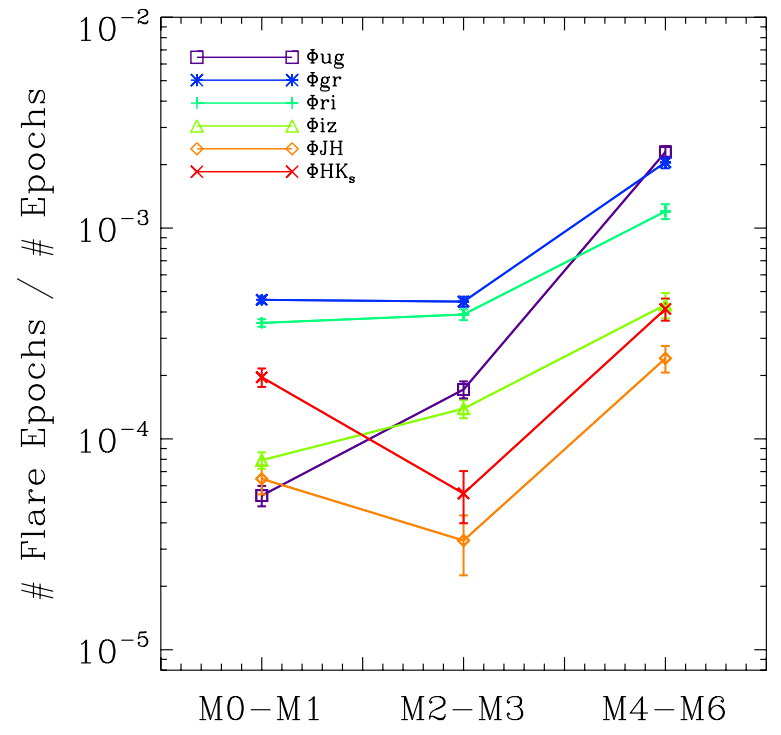

Figure 6. Fraction of flaring epochs, defined as the number of good epochs above the $\Phi$ threshold divided by the total number of good epochs, for each filter combination and spectral-type bin.

(A color version of this figure is available in the online journal.)

The number of flaring epochs for each filter combination was very small compared to the total number of observations. Previous studies such as K09 have shown that flare rates for $\mathrm{M}$ dwarfs increase as a function of spectral type. To examine this trend, we grouped our sample into three spectral-type bins (M0-M1, M2-M3, M4-M6). The ratio of flare candidate epochs to the total number of epochs for each bin is shown in Figure 6. As expected, all the optical SDSS filter combinations show an increasing numbers of flares with later spectral type (K09). There were considerably fewer flare epochs detected in the NIR passbands, although the $\Phi_{J H}$ and $\Phi_{H K}$ flare rates fall within the range of the SDSS data in Figure 6. This is likely due to the weak signatures of flares in the NIR, as discussed in Section 5, and the lower photometric accuracy for these stars in the 2MASS data. No apparent trends of $\Phi$ with air mass, photometric error, or photometric quality flags were seen in the final Cal-PSWDB candidate flare sample. Table 2 presents the total numbers of
Table 2

Summary of Flare Candidates in Each Spectral Type Bin per Wavelength Pair

\begin{tabular}{lcccc}
\hline \hline$\Phi$ & Spectral Type & Candidate Flares & Number Epochs & Flare Fraction \\
\hline$u g$ & M0-M1 & 81 & $1.5 \times 10^{6}$ & $5.4 \times 10^{-5}$ \\
$u g$ & M2-M3 & 116 & $6.8 \times 10^{5}$ & $1.7 \times 10^{-4}$ \\
$u g$ & M4-M6 & 262 & $1.1 \times 10^{5}$ & $2.3 \times 10^{-3}$ \\
$g r$ & M0-M1 & 722 & $1.6 \times 10^{6}$ & $4.6 \times 10^{-4}$ \\
$g r$ & M2-M3 & 322 & $7.2 \times 10^{5}$ & $4.5 \times 10^{-4}$ \\
$g r$ & M4-M6 & 251 & $1.2 \times 10^{5}$ & $2.1 \times 10^{-3}$ \\
$r i$ & M0-M1 & 567 & $1.6 \times 10^{6}$ & $3.6 \times 10^{-4}$ \\
$r i$ & M2-M3 & 283 & $7.3 \times 10^{5}$ & $3.9 \times 10^{-4}$ \\
$r i$ & M4-M6 & 149 & $1.2 \times 10^{5}$ & $1.2 \times 10^{-3}$ \\
$i z$ & M0-M1 & 125 & $1.6 \times 10^{6}$ & $8.0 \times 10^{-5}$ \\
$i z$ & M2-M3 & 100 & $7.2 \times 10^{5}$ & $1.4 \times 10^{-4}$ \\
$i z$ & M4-M6 & 53 & $1.2 \times 10^{5}$ & $4.3 \times 10^{-4}$ \\
$J H$ & M0-M1 & 41 & $6.3 \times 10^{5}$ & $6.5 \times 10^{-5}$ \\
$J H$ & M2-M3 & 10 & $3.0 \times 10^{5}$ & $3.2 \times 10^{-5}$ \\
$J H$ & M4-M6 & 48 & $2.0 \times 10^{5}$ & $2.4 \times 10^{-4}$ \\
$H K$ & M0-M1 & 99 & $5.1 \times 10^{5}$ & $1.9 \times 10^{-4}$ \\
$H K$ & M2-M3 & 13 & $2.4 \times 10^{5}$ & $5.5 \times 10^{-5}$ \\
$H K$ & M4-M6 & 69 & $1.7 \times 10^{5}$ & $4.1 \times 10^{-4}$ \\
\hline
\end{tabular}

flare candidates in each band for the three spectral-type bins. No spectroscopy for our candidate NIR flare stars was available to determine their level of $\mathrm{H} \alpha$ activity.

A control sample from both Cal-PSWDB and Stripe 82 was created by downloading light curves for earlier spectral-type stars, which have a much lower intrinsic flare rate. We obtained the same number of light curves as in Section 2 for stars with $0.5 \leqslant(g-i) \leqslant 1.4$, corresponding to spectral types G0-K5 (Covey et al. 2007). The same quality cuts were applied, and $\Phi$ values calculated. These light curves produced more than an order of magnitude fewer flare candidates in every band and no very high $\Phi$ epochs $(\Phi>100)$. We are confident that our $\mathrm{M}$ dwarf flare candidate sample is not due to systematic contamination in our data.

\subsection{Flare Luminosities}

To compare our results in many photometric bands to those of previous studies, we must transform the flares from apparent 


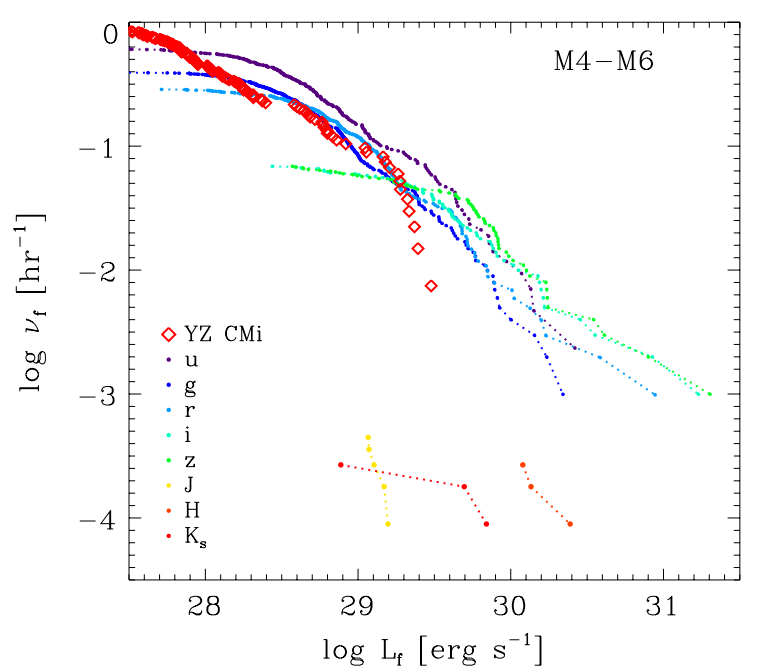

Figure 7. Cumulative flare frequency distributions as a function of observed luminosity in each passband for a sample spectral-type bin. Errors in luminosity are dominated by photometric errors and assumptions in the distance. Incompleteness in recovering flares at the low-luminosity end results in the flattening seen clearly in each of the SDSS bands.

(A color version of this figure is available in the online journal.)

magnitude enhancements to luminosities. The apparent magnitudes for every epoch and filter in our time-domain Stripe 82 and Cal-PSWDB samples were first converted to observed flux, in units of erg s $\mathrm{s}^{-1} \mathrm{~cm}^{-2} \mu \mathrm{m}^{-1}$, using the calibrations from Ivezić et al. (2007) and Cohen et al. (2003), respectively. To estimate the quiescent luminosity for each epoch, we obtained distance estimates for our stars from the $\left(\mu_{r}, r-z\right)$ photometric parallax relation of Bochanski et al. (2010), using the $(r-z)$ color from DR7. Errors in the computed luminosities are dominated by the distance uncertainties.

Many previous studies have shown that the frequency of flares decreases as a function of increasing flare energy (e.g., Lacy et al. 1976) or flare luminosity (K09). For each bandpass we selected the epochs identified as flare candidates in Table 1 and found a cumulative distribution in flare luminosity. The frequency per hour that these flares were observed was calculated by dividing the cumulative number distribution by the total observing time for each filter, giving the flare frequency distributions plotted in Figure 7. These show for each filter, $f$, the number of flares per hour of observation, $v_{f}$, as a function of observed luminosity, $L_{f}$. The YZ CMi flare frequency distribution from Lacy et al. (1976) is shown and corresponds well with our optical distributions. Dedicated flare monitoring studies, such as Hilton et al. (2010a), can correct the low-luminosity flaring frequencies for incompleteness due to changing observing conditions. Such corrections are beyond the scope of this work.

The optical (ugriz) distributions are very similar, indicating that flares produce smooth continuous variations in these bandpasses. As with Figure 6, the $J H K_{s}$-band flare frequency distributions in Figure 7 are highly undersampled. NIR flares were recovered more than two orders of magnitude less frequently than for SDSS optical colors for the M4-M6 bin.

The turnover at low luminosities seen in the SDSS filters is due to incompleteness in the low-luminosity flare census. To explore whether the observed power-law distributions (from $\log L_{f}=29$ to $31 \mathrm{erg} \mathrm{s}^{-1}$ ) extend to even lower luminosity flares than our $\Phi$ thresholds recover, we moved the $\Phi^{+}$detection thresholds to higher and lower values in the $u$ band. In Figure 8 the power-law slope appears to extend to much

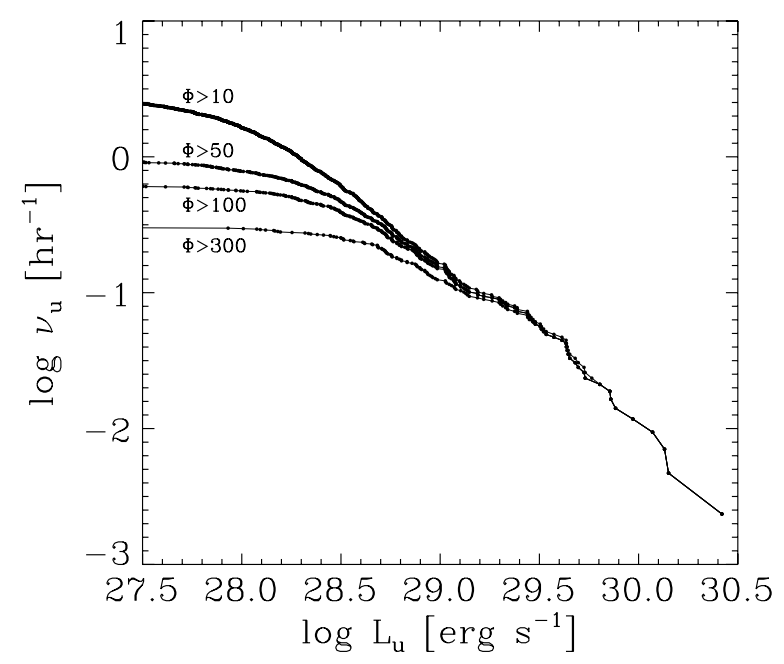

Figure 8. Cumulative flare frequency distribution, as in Figure 7, for the $u$ band using four different $\Phi$ thresholds to select flare candidate epochs. Lower thresholds allow more contaminating epochs into our flare distribution, but the power-law slope seen at luminosities above $\sim 10^{29} \mathrm{erg} \mathrm{s}^{-1}$ remains the same.

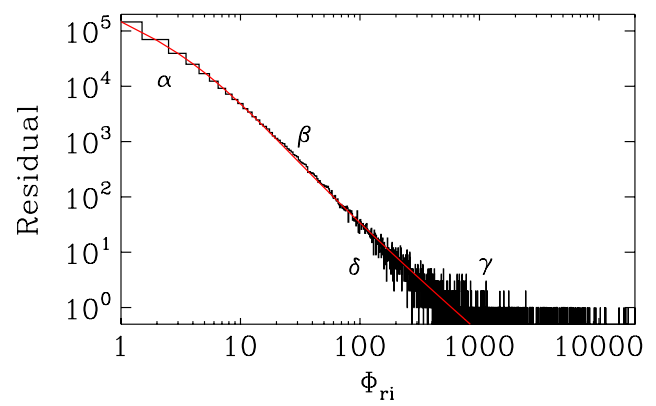

Figure 9. $\Phi_{r i}$ residual (black), and the corresponding fit (red) defined in Equation (2).

(A color version of this figure is available in the online journal.)

lower luminosities as a result of the lower $\Phi$ thresholds. We recovered many additional flare candidate epochs, but with a much higher rate of false positives. The high-luminosity end of Figure 8 (above $\log L_{u} \sim 29.1 \mathrm{erg} \mathrm{s}^{-1}$ ) is insensitive to the choice of $\Phi$ threshold.

\subsection{Residual $\Phi$ Distributions}

The $\Phi$ distributions in Figure 5 indicate that there are many more epochs in the $\Phi^{+}$distribution than in the $\Phi^{-}$, showing that the two bandpasses that make up each $\Phi$ distribution preferentially increased in flux together. The excess between the $\Phi^{+}$and $\Phi^{-}$is also evident at values of $\Phi$ well below the flare candidate thresholds. By subtracting the $\Phi^{-}$from the $\Phi^{+}$ distributions for each of the six filter combinations in Figure 5, we are left with residual $\Phi^{+}$distributions, as shown in Figure 9 for $\Phi_{r i}$. These residual distributions follow a roughly powerlaw profile below the $\Phi^{+}$thresholds, with a turnover at low $\Phi^{+}$ amplitudes. We fit the residual distributions using the equation

$$
\log _{10} Y=\alpha+\left(\beta \log _{10} X\right)\left(10^{-\gamma /(X+\delta)}\right),
$$

where $Y$ is the $\Phi^{+}-\Phi^{-}$residual, $\alpha$ is the peak amplitude for the residual, $\beta$ is a power-law slope at larger luminosities, $\delta$ is the turnover $\Phi$ between the power law and the flattening at low $\Phi, \gamma$ is the exponential plateau slope at low $\Phi$, and $X$ is the $\Phi^{+}$value. Figure 9 also illustrates where in the residual distribution each of the fit coefficients constrains. This functional form was chosen to simultaneously fit the residual power-law tail at large $\Phi$ and the 


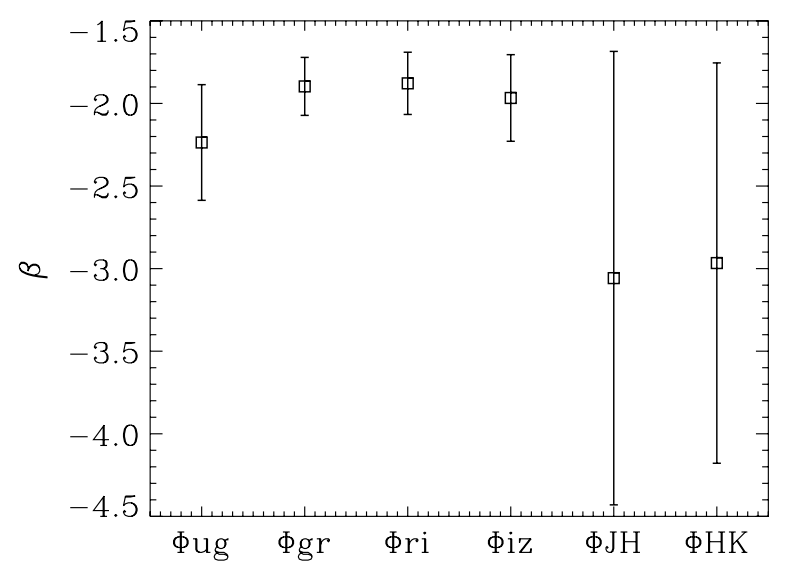

Figure 10. Slope of the residual $\Phi$ distributions, as defined in Equation (2), for each filter combination. The steeper slope at increasing wavelength is due to the lower intrinsic rate of variability within the photometric errors.

turnover at low $\Phi$ values due to random noise dominating. The power-law coefficient $\beta$ is plotted for each of the distributions in Figure 10. The $\beta$ values ranged between -1.9 and -3.1 . The higher slope in $\beta$ in the $\Phi_{g r}$ fit may be due to the better photometric precision in the $g$ and $r$ bands, compared to the $u$ band, allowing those filters to be more sensitive to flares below the $\Phi$ threshold. The Cal-PSWDB $\Phi_{\mathrm{HKs}}$ conversely shows the smallest observed residual, again suggesting that these longer wavelength filters are less affected by intrinsic stellar variability. However, all the fits are consistent with a value of $\beta \approx-2$ within the errors.

The physical cause of this power-law slope, $\beta$, at $\Phi$ values less than our flare cutoff threshold, may be the extension of the increasing flare frequency at lower luminosities. Lacy et al. (1976) showed that the flare frequency distribution has a powerlaw shape, with a turnover at lower energies due to confusion with the quiescent variability, and a falloff at higher energies due to incompleteness. Their flare detection extended to lower energy flares than our analysis was sensitive to. This trend has been verified for $\mathrm{M}$ dwarf flares more recently by K09 and Hilton et al. (2010b).

There is not a direct transformation to compare between our observed $\Phi$ residual distributions and the flare frequency distributions calculated by Lacy et al. (1976) and others. Our residual $\Phi$ distributions contain all the $M$ sub-types combined, for stars across a large range of distances. Hilton et al. (2010a) have shown that the flare frequency distribution slopes are similar, though not constant, for different spectral-type bins and between active and inactive stars.

Robinson et al. (1995, 1999) found that "microflares," very frequent low-energy rapid flares, were detectable using Hubble Space Telescope (HST) for two very active M dwarfs. By comparing the distribution of fluxes about the median quiescent value in their light curves, the microflares, which are of too low amplitude to be detected individually, could be detected statistically. They found that these microflare events followed a power-law distribution that was steeper than that of the larger resolved flares previously detected. Thus, there are even greater numbers of microflares than would be predicted by simply extending the power-law flare frequency to lower energies.

Our method of finding the residual $\Phi$ distribution yields a similar result, by determining an asymmetry in the light curves via the $\Phi$ statistic due to excess flux enhancements. However, we caution that we are not detecting the extremely low amplitude microflares seen in the HST observations from
Robinson et al. (1995). Instead, the residual power in the $\Phi$ distributions is likely a result of the underlying cacophony of low-amplitude flares that are below our single-epoch detection limits in each bandpass, but which would be visible in highprecision continuous monitoring programs. It is especially notable that the significant asymmetric distributions were seen in all wavelengths studied $\left(u-K_{S}\right)$, although we are sampling very different contrasts with the underlying quiescent stellar spectrum, which supports our interpretation of these events as flares. While the flare frequency distributions in Figure 7 are undersampled for the NIR filters, the residual epochs with excess flux seen in the $\Phi_{J H}$ and $\Phi_{\mathrm{HKs}}$ in Figure 5 and their power-law slopes in Figure 10 provide a statistically significant detection of small-amplitude flux enhancements.

\section{OPTICAL AND NIR FLARE MODEL}

We have employed a semi-analytical two-component flare model, as first advocated by Kunkel (1970), to explore the completeness of our search for flares in every filter and to compare the observed flare luminosities between filters. We extend the Kowalski et al. (2010) model, which was used in blue optical wavelengths to red optical and NIR bandpasses.

In order to simulate flux enhancements due to flares in each filter using the two-component model, we built quiescent template M dwarf spectra in the optical and NIR. We utilized flux-calibrated spectra from the IRTF stellar catalog (Cushing et al. 2005; Rayner et al. 2009) for stars with spectral-type M0 through M6. The M0 through M6 SDSS templates from Bochanski et al. (2007b) were used for most of the optical regime. The IRTF and Bochanski spectra were normalized and joined at $8500 \AA$ A. Since the Bochanski optical templates only go as short as $3825 \AA$, to fully cover the M dwarf spectra in the $u$ band we used templates from Pickles (1998). As these are lower resolution than the Bochanski templates, we used them only from $2900 \AA$ to $5000 \AA$. The Pickles and Bochanski templates are both published in normalized flux units, while the flux-calibrated IRTF data we used in the NIR are in observed flux. We found distances to each of the IRTF M dwarf standard stars from Jenkins (1952), van Altena et al. (1995), van Leeuwen (2007), and Jenkins et al. (2009) using the SIMBAD database, and standard radii from N. Reid \& S. L. Hawley (2000) to convert the observed flux into surface luminosity for each star in the NIR. The normalized optical templates were then scaled to match at $8500 \AA$, and resultant optical/NIR spectral energy distributions for the M0 through M6 sub-types are shown in Figure 11.

The lower panel of Figure 12 presents our M3 spectrum in arbitrary flux units in black, with the ugrizJHK $K_{s}$ transmission curves from Fukugita et al. (1996) and Cohen et al. (2003) shown for reference. The overall flare SED shape was defined by a $10,000 \mathrm{~K}$ blackbody continuum. Previous studies have found temperatures as low as $\sim 8500 \mathrm{~K}$ (Hawley \& Fisher 1992) for flares, while Kowalski et al. (2010) found evidence for temperatures as high as 13,000 K. Fuhrmeister et al. (2008) fit a blackbody temperature of $20,000 \mathrm{~K}$ during the brief impulsive phase of an enormous flare on $\mathrm{CN}$ Leo. These temperatures rapidly declined, however, with values around 7000-10,000 K for the remainder of the flare event.

Shortward of $3646 \AA$, we added a second component of $\mathrm{H}$ Balmer continuum line emission, which was computed from the Allred et al. (2006) radiative hydrodynamic M dwarf atmosphere model. The Balmer continuum was scaled to have 10 times larger surface area coverage than the blackbody component. 


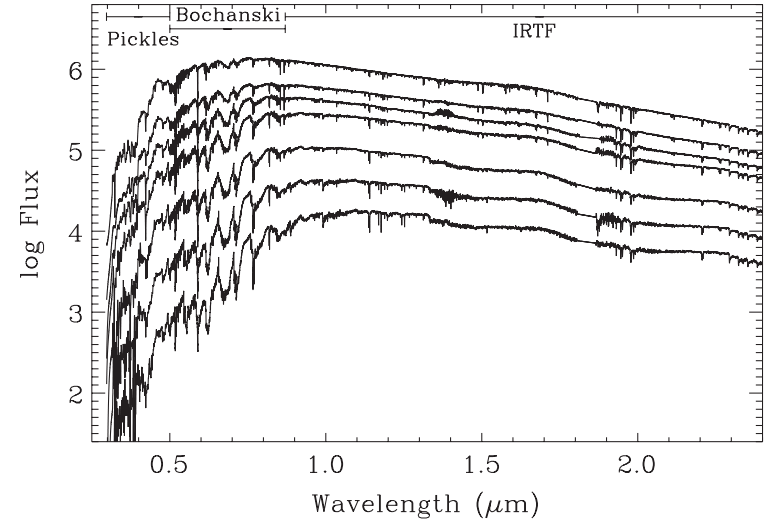

Figure 11. Combined $2900 \AA$ to $2.5 \mu \mathrm{m}$ spectral energy distributions for our M0-M6 templates. The ranges in wavelength over which each of the template sources contributes are shown at top.

The 10:1 scaling was approximately an average of those used by previous studies, with Kowalski et al. (2010) using values from 3:1 to $16: 1$. Our idealized model does not include many detailed emission mechanisms that would alter these fluxes, such as line emission from other elements, or other levels of hydrogen. Using measured spectral line emission from M dwarf flares in the NIR from Schmidt (2012), we estimate that line emission would contribute an order of magnitude less than our predicted blackbody continuum emission in $J H K_{s}$ bands and is therefore negligible for our analysis. Continuum emission from higher levels of hydrogen in the flare atmosphere and effects from chromospheric back-warming such as discussed in $\mathrm{Xu}$ et al. (2006) likely provide the greatest changes to the red optical and NIR flare SED.

To investigate the effect of the flare model, we scale it by a surface coverage fraction, ranging from $5 \times 10^{-7}$ to 0.5 , and add it to the M0-M6 template spectra. The photometric response to flares in each filter was then calculated by convolving the SDSS and 2MASS filter transmission curves (Fukugita et al. 1996; Cohen et al. 2003) to the M0-M6 model spectra with and without the flare. In Figure 13 we show the transformations between the $\Delta u$-band and the $\Delta\left(\right.$ grizJHK$\left._{s}\right)$-band amplitudes for all the flare models we ran.

The top panel of Figure 12 shows the changes in each photometric bandpass as computed by our model for a flare on an M3 star with $1 \%$ surface coverage for the Balmer continuum and $0.1 \%$ coverage for the blackbody component. The resulting increases in flux for the ugrizJHK $K_{s}$ bands were approximately $(2.7,0.62,0.19,0.06,0.03,0.01,0.01,0.01) \mathrm{mag}$, respectively.

The extremely blue response of the flare is due to a contrast effect between the intrinsically blue flare model and the underlying red stellar spectrum. Since the M3 spectrum is much redder than the flare model, even large-amplitude flares do not give a strong signal in the reddest filters. However, K09 recovered a very bright flare in the Stripe 82 sample, visible in all five passband light curves, on an active M7 star. This star was removed from our analysis as it had too late of a spectral type. The flare had magnitude excursions of $(5.50,3.07,2.34,0.77,0.29)$ for the ugriz bands, respectively. Our M6 model, scaled to match the $u$-band excursion, predicted ugriz magnitude excursions of $(5.5,2.5,1.2,0.3,0.1)$. In the 2MASS $J H K_{s}$ filters we predicted excursions of approximately $(0.03,0.01,0.01)$. The coverage for this simulated flare was $0.06 \%$ of the stellar surface. As the two-component model does not include detailed effects, as discussed above, the model excursions agree with the observations to within a factor of two in flux. The K09 flare may also have evolved in temperature over the course of the SDSS observations (Fuhrmeister et al. 2008). We allowed the blackbody temperature within our model to vary from $8000 \mathrm{~K}$ to $13,000 \mathrm{~K}$. Lower temperatures yielded only a marginally improved fit for the $r$ band and required a larger effective surface area for the flare.

\section{MODEL FLARE RECOVERY}

Using our flare model with the M3 spectral template, we investigated the efficiency of recovering flares from our sample with the $\Phi$ statistic. We first computed the sigma-clipped mean photometric error profiles as a function of observed magnitude for each of the $u g r i z J H K_{s}$ bands in increments of $0.1 \mathrm{mag}$,

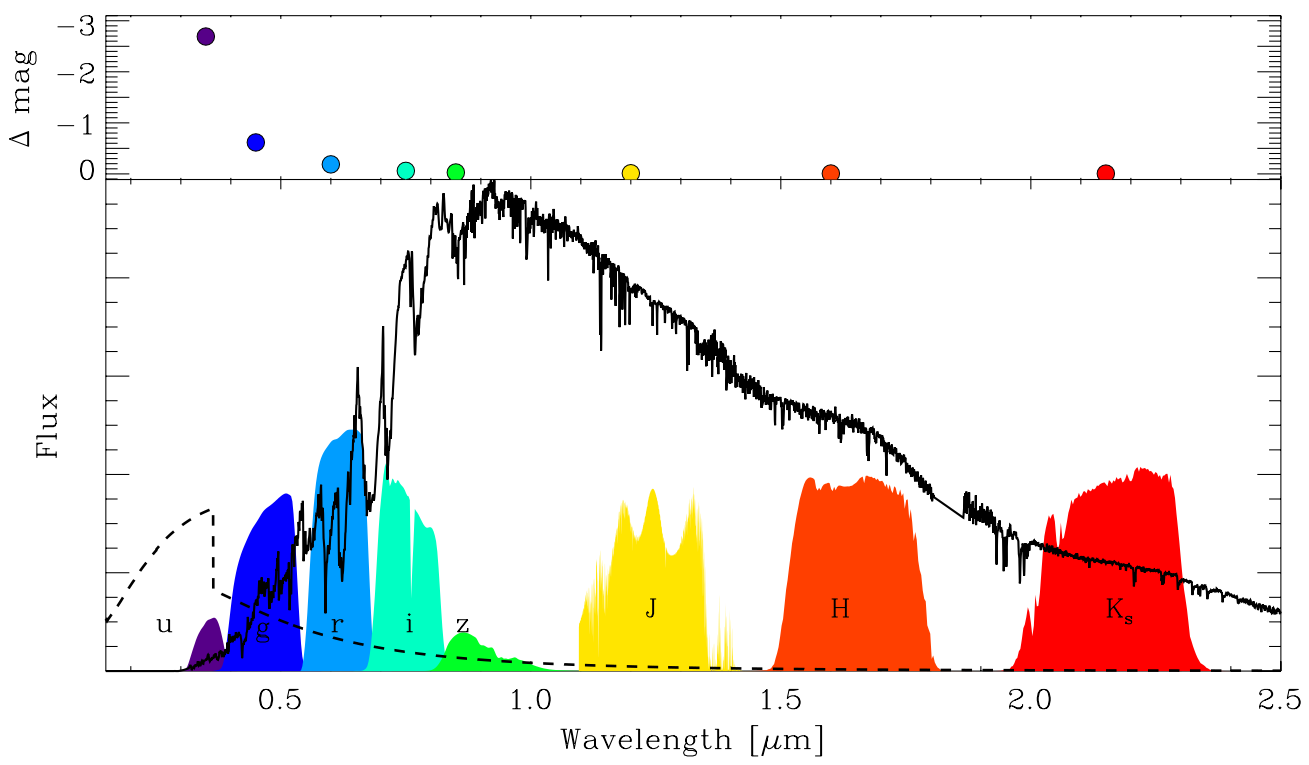

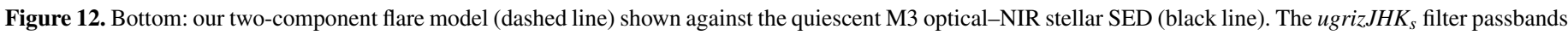
are shown for reference. Top: the resulting change in magnitude for each filter from a sample flare with $1 \%$ surface coverage on an $\mathrm{M} 3$.

(A color version of this figure is available in the online journal.) 


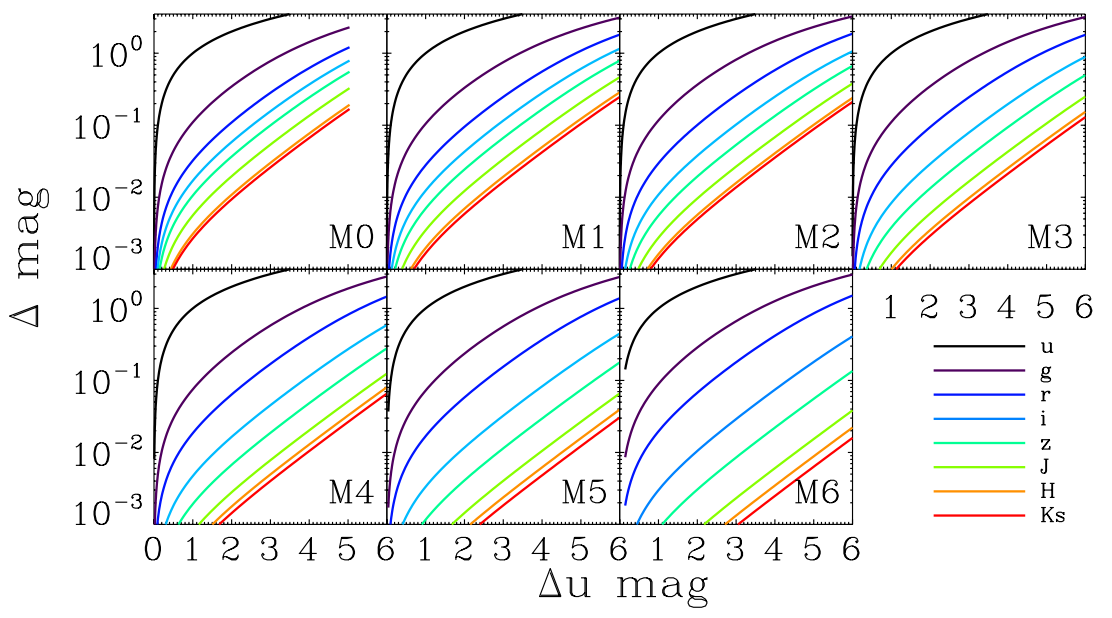

Figure 13. Transformation between the predicted $u$-band response and the grizJHK $K_{s}$-band response for each spectral-type template. (A color version of this figure is available in the online journal.)

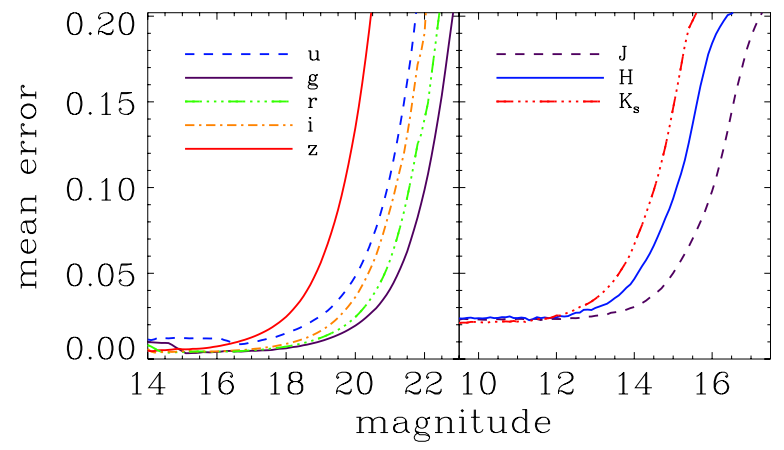

Figure 14. Mean photometric error as a function of apparent magnitude for all the Stripe 82 and Cal-PSWDB measurements in our database.

(A color version of this figure is available in the online journal.)

shown in Figure 14. For each of the M3 stars in our NIR timedomain sample, we simulated 200 artificial flares using our model from Section 5, with a logarithmically increasing surface area coverage fraction from $10^{-7}$ to $10^{-1}$. The mean error profiles were then used to select an appropriate photometric error for the resultant apparent magnitudes of each simulated flare in all eight passbands. Finally, we calculated the $\Phi$ values for each simulated flare on every star.

The result was a " $\Phi$ profile," which was a function of both the quiescent apparent magnitude of the star and the flare amplitude. These represent the two competing physical quantities, the distance to the star and the size of the flare, which conspire to limit our detection of small flares or flares on distant stars. This envelope is shown in Figure 15 as a function of quiescent magnitude on the $x$-axis, with $\Phi$ contour values increasing from black to red. The $\Phi$ profile was smoothed with a kernel of 0.1 mag. Because flares are typically characterized by their enhancement of blue flux, the corresponding predicted $u$-band excursion from our model is shown on the right axis in blue for the grizJHK $K_{s}$ bands.

Using our described $\Phi$ thresholds from Section 4, we found that small flares in the $u$ band $(\Delta u \sim 0.5 \mathrm{mag}$ ) would be recovered for every star in the sample with a quiescent magnitude of $u \leqslant 20.5$. The completeness is a direct function of the photometric error profile, which parallels the $\Phi$ threshold curves shown in Figure 15. Interestingly, note that in the griz bands in Figure 15, the $\Phi$ profiles "turn up" for bright stars, since the error profiles begin to increase as stars approach the saturation limit for the SDSS. Consider a star with a moderate quiescent luminosity in the $u$ band (e.g., $u \sim 17$ ). A very large flare such as those observed in Hawley \& Pettersen (1991) and Kowalski et al. (2010) would result in an observed $u$ band magnitude too bright for the SDSS photometry and would therefore be excluded from our analysis. This would be possible in the $g$ and even $r$ band for bright stars with very large flares. Incompleteness is therefore also expected at the brightest flare luminosities, although these events are rare with duty cycles estimated to be $\sim 1$ per month on most active stars (Hilton et al. 2010b).

The error profiles in the $g, r$, and $i$ filters plotted in Figure 14 are best suited to monitor small-amplitude variations such as eclipses and spot modulation. Figures 15 and 13 clearly show that we are only completely sampling flares larger than $\Delta u \sim 2$ in these bands for most of our sample, and thus the $u$ band is the most suitable to search for the frequent low-amplitude flares in these stars.

As expected from the model discussion above, only very large flares would be detectable in the NIR passbands for the brightest stars in our sample using the 2MASS photometric error profile. Our model predicts that a flare with a nearly $\sim 5 \mathrm{mag}$ increase in the $u$ band would be required to detect any significant increase in flux in the Cal-PSWDB for a nearby M3-type star. No simulated flares on M3 stars showed enough increase in flux to register as a $\Phi=50$ response in the $J H K_{s}$ bands, however. The flare emission in the NIR must therefore be greatly influenced by physics not included in our two-component model, and definitive simultaneous detection of flares in the NIR and optical is needed to calibrate this model.

\subsection{Correcting for Efficiency}

Varying observing conditions can introduce small changes in the photometric zero point for the Cal-PSWDB sources (Plavchan et al. 2008b) and increase the photometric error for both Stripe 82 and Cal-PSWDB data. This in turn will decrease our efficiency in recovering flares at low luminosities. With our model for flare color as a function of both spectral type and flare amplitude (Figure 13), we estimated the minimum amplitude flare that could have been observed for every epoch in each filter. For each non-flaring epoch in our sample we generated flares with a range of amplitudes using our flare model. This flux 


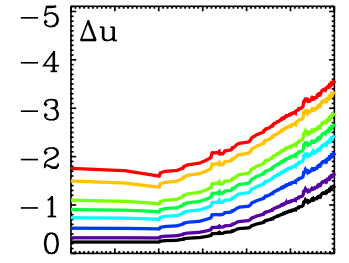

17181920212223

$\mathrm{u}$

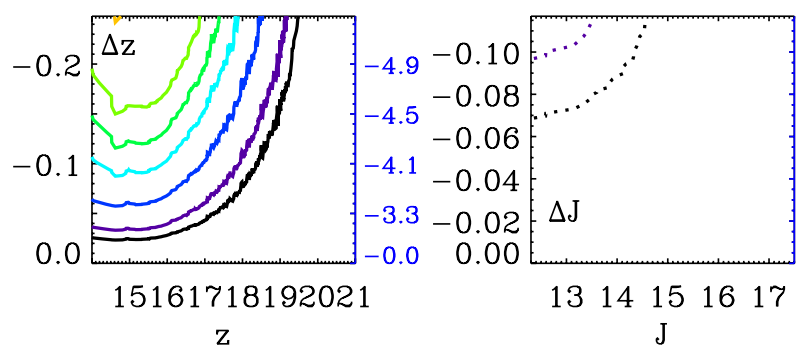

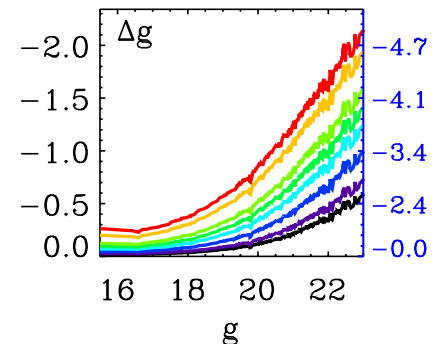
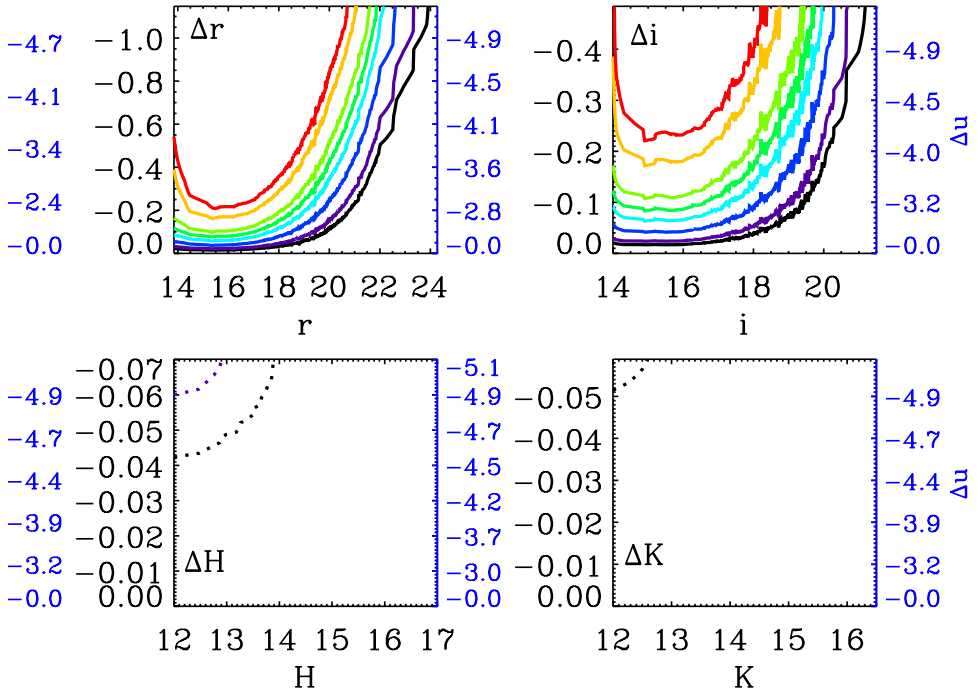

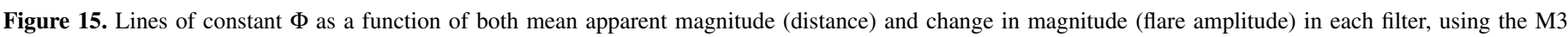

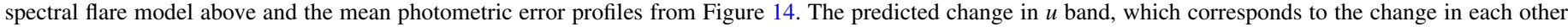

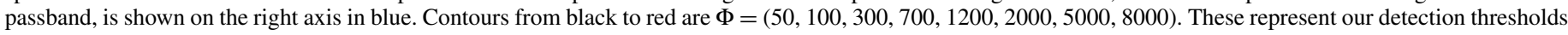
for a given flare amplitude as a function of apparent magnitude in these surveys. The dotted-line threshold contours for $2 \mathrm{MASS}$ filters are $\Phi=5$ and 10 .

(A color version of this figure is available in the online journal.)

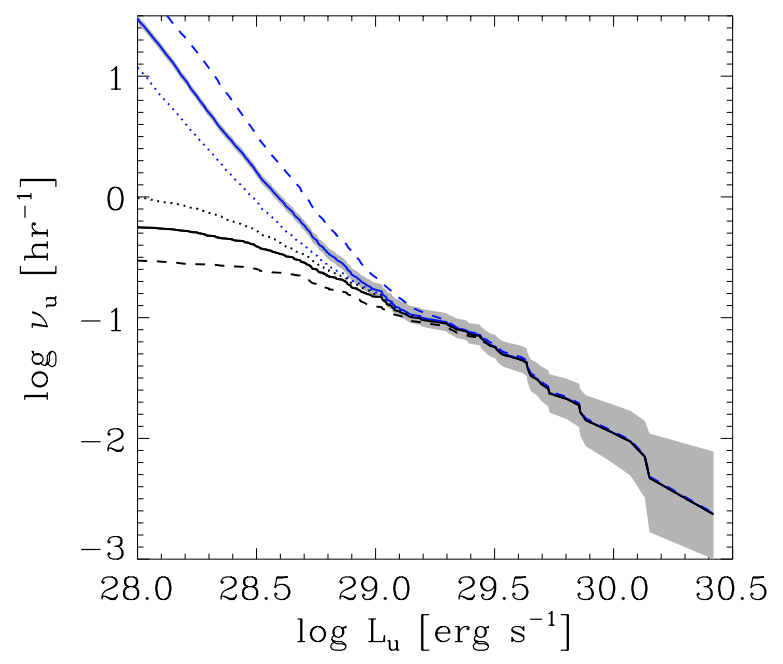

Figure 16. $u$-band M4-M6 cumulative flare frequency distributions (black lines) for three different $\Phi_{u}$ thresholds $\left(\Phi_{u}>300\right.$-dashed; $\Phi_{u}>100$ - solid; $\Phi_{u}>30$ - dotted). Efficiency-corrected distributions are shown (blue lines), with the Poisson error profile for the $\Phi_{u}>100$ highlighted in gray.

(A color version of this figure is available in the online journal.)

enhancement was added to the measured quiescent flux, and the photometric uncertainty decreased based on our error profiles. The flare amplitude necessary to achieve the $\Phi$ threshold values was measured, and a limiting flare luminosity $\left(L_{\mathrm{lim}}\right)$ that varies based on observing conditions was assigned. The efficiencycorrected flare frequency distribution was calculated as $v\left(L_{n}\right)=$ (flares with $\left.L \geqslant L_{n}\right) /\left(\right.$ epochs with $\left.L_{\lim } \leqslant L_{n}\right) / t_{\text {exposure }}$.

We present the observed and efficiency-corrected $u$-band flare frequency distributions in Figure 16 for three different $\Phi_{u}$ thresholds. The efficiency correction increases the rate of flares per hour at faint luminosities by more than an order of magnitude. The Poisson error profile for the corrected $\Phi_{u}>100$ distribution is dominated at large luminosity by the small numbers of flares detected. The error profile increases slightly at low luminosity due to the decreasing number of quiescent epochs with suitable $L_{\text {lim }}$.
The efficiency-corrected distributions in Figure 16 begin to diverge from those measured at $\log L_{u} \sim 29 \mathrm{erg} \mathrm{s}^{-1}$, suggesting that our observed sample is complete down to this luminosity. These corrections, applied to our sparsely sampled data, have many systematic dependencies, such as spectral-type assignment and model flare colors. This is the first attempt to efficiency-correct the measured flare rate for sparsely sampled light curves of many stars. A dependence on the $\Phi$ threshold still remains, indicating that our prescription did not fully characterize the inefficiency of flare recovery. Dedicated monitoring programs, such as Hilton (2011) and Tofflemire et al. (2012), will be able to estimate incompleteness in time-resolved flare detection due to changing observing conditions with much greater precision.

\subsection{Optimal NIR Flare Detection}

Our analysis has extrapolated a well-known two-component flare model into a new wavelength regime, and we therefore provide a prediction for the "best case" to detect $\mathrm{M}$ dwarf flares in the NIR. An enhancement of $\Delta J \geqslant 0.01$ magnitudes is easily observable for many nearby $\mathrm{M}$ dwarfs with present-day groundbased differential photometry (e.g., Tofflemire et al. 2012). Using the $\Delta u$-to- $\Delta J$ flare amplitude transformations shown in Figure 13, we estimated the $\Delta u$ enhancement for each spectral type required to achieve $\Delta J=0.01$. For spectral types M0-M6 this yielded a $\Delta u=(1.4,1.8,2.0,2.5,3.3,4.0,4.5) \mathrm{mag}$. The changing shape in the underlying stellar SED in the NIR indicates that $J$-band flares are detectable from smaller $\Delta u$ flares at earlier spectral types.

The frequency of flaring has been previously found to increase with spectral type, such as in our Figure 6 . The characteristic lifetime of magnetic activity is also highly correlated with spectral type (West et al. 2008), and later spectral-type M dwarfs are expected to show not only more but larger amplitude flares (Hilton 2011). This highlights the contrasting effects at work in detecting broadband NIR flares: persistently active M0 stars that show large-amplitude flares are exceedingly rare, as compared to those at later spectral types. The higher bolometric luminosity 


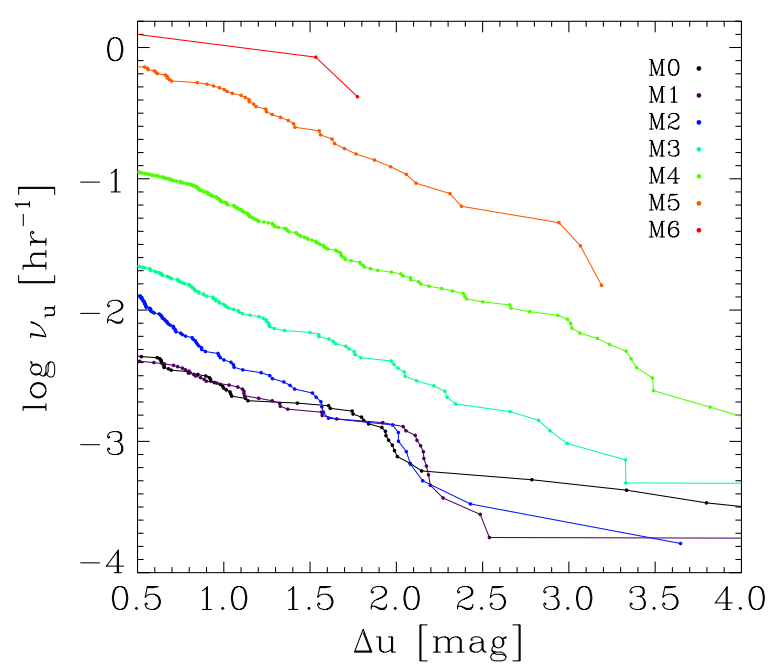

Figure 17. Observed flare frequency distribution for $u$-band flares at each spectral type. Each distribution was fit with a linear polynomial in the range $0.4 \leqslant \Delta u \leqslant 3.0$.

(A color version of this figure is available in the online journal.)

of earlier spectral-type stars additionally means that much larger energy flares are needed to achieve these large amplitudes.

Figure 17 shows the observed $u$-band flare frequency distribution for each spectral-type bin as a function of $\Delta u$ magnitude. The total rate of flaring increased with spectral type, and the slopes were very similar for all the distributions, in agreement with results from Hilton (2011). A linear fit was found for each $\log _{10} v(\Delta u)$ distribution between $0.4 \leqslant \Delta u \leqslant 3.0$ to avoid falsepositives at the low-amplitude end and incompleteness at high $\Delta u$ amplitudes. The predicted frequency at which we expect to observe a $\Delta J \geqslant 0.01 \mathrm{mag}$ flare, from the corresponding $\Delta u$ amplitudes, at each spectral type was then calculated from these fits and is shown in Figure 18. The optimal spectral type to search for these NIR flare detections is in our latest-type stars, M6. The slight upturn at M0 is due to the more favorable color contrast between the flare and the star. The overall trend from M1 to M6, however, shows that the strongest effect for all spectral types is the total flaring duty cycle. This provides one possible reason that the recovered flare rates are higher than expected for M0-M1 stars in Figure 6. A new method for calculating the duty cycle of a given amplitude excursion, known as the Magnitude Likelihood Distribution, has been recently put forward by Hilton (2011). Using these Magnitude Likelihood Distributions, we predict a peak duty cycle of $\sim 3 \times 10^{-4}$ for the magnetically active M4 and M5 stars. For the $54 \mathrm{~s}$ exposures of SDSS, this is equal to a frequency of $v \approx 3 \times 10^{-2} \mathrm{hr}^{-1}$, which is consistent with our estimation. We would encourage future NIR flare observers to monitor bright/nearby targets in the latest spectral types possible, at least M4 or later. Promising stars might include GJ 1061, CN Leo (Wolf 359), and especially the highly active YZ CMi.

\section{SUMMARY AND CONCLUSIONS}

We have shown that the signatures of flares from M dwarfs are not confined to the bluest bandpasses in large-scale photometric surveys. There is significant response from medium to large flares in gri bands, and the largest flares are observable in $z$ and in some cases into the NIR passbands. This is the first statistical characterization of $\mathrm{M}$ dwarf flares in the NIR regime.

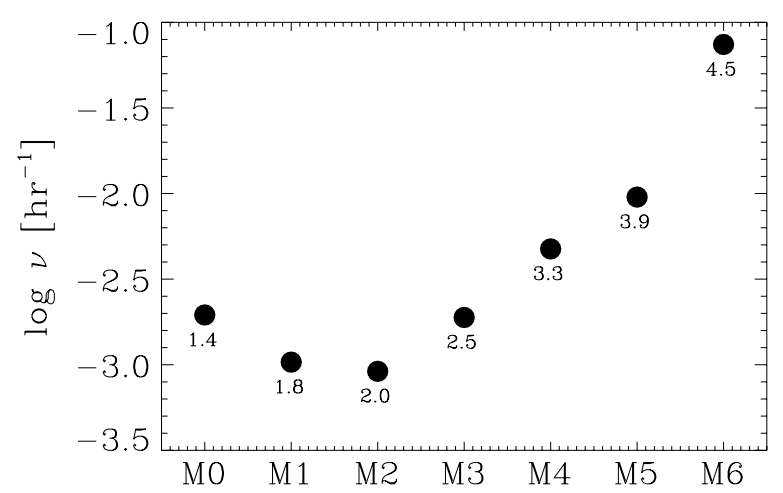

Figure 18. Rate per hour at which a $\Delta J \geqslant 0.01 \mathrm{mag}$ amplitude flare is expected to be seen on each spectral type, based on our model and measured $u$-band flare rates. The numbers below each point are the $\Delta u$ amplitudes that our flare model predicts from a $\Delta J=0.01 \mathrm{mag}$ flare at each spectral type.

A self-consistent result between all eight filters shows that while the frequency of flares decreases with increasing luminosity in all filters, the total number of flares recovered decreases with wavelength. This suggests that, like our simplified flare model, the underlying SED for flares of all sizes is intrinsically blue. Furthermore, the total rate of flares in all optical bands appears to increase with increasing spectral type, but low-number statistics prevent us from robustly exploring this distribution at NIR wavelengths. The slope of the flare frequency distribution is found to be insensitive to the flare detection threshold below $\sim 10^{29} \mathrm{erg} \mathrm{s}^{-1}$.

We have also presented an implementation of the twocomponent spectral flare model from Kowalski et al. (2010) to make predictions of the red-optical and NIR response of flares in $\mathrm{M}$ dwarfs. These conversions will be useful in future broadband photometric studies of $\mathrm{M}$ dwarfs to predict the contamination from flares across a wider wavelength space. Flares in the $J$ band are predicted to be detected most frequently in spectral types from M4 to M6, with rates of $\sim 2 \times 10^{-2} \mathrm{hr}^{-1}$.

Medium-sized flares larger than $0.5 \mathrm{mag}$ in $u$ were found to occur at a rate of one per few thousand SDSS exposures for $\mathrm{M}$ dwarfs. Low-amplitude flare rates are limited by the photometric precision of the survey in question, and LSST will detect even smaller amplitude flares from these stars and will increase the rate at which variability from these sources is detected. LSST will also employ a $y$-filter, with a central wavelength between that of $z$ and $J$. Using the " $y 4$ " filter curve for LSST, our two-component flare model from Figure 12 predicts a flare amplitude of $\Delta y 4 \sim 0.02 \mathrm{mag}$. The increased photometric sensitivity will therefore allow LSST to routinely detect large flares in this NIR bandpass for bright stars, providing useful constraints on the SED structure of flares at long wavelengths.

Finally, we have shown that the intrinsic SED of a flare, convolved with the photometric characteristics of a given survey, can greatly affect the sample of flares recovered. The cadence and integration times will also affect the recovery of flares. Short exposures such as in 2MASS capture the nearinstantaneous luminosity of an event, while longer exposure times may encapsulate entire flare events. We anticipate a day when this problem can be inverted, using orders of magnitude larger samples of flare candidate epochs in many bands, as well as spectra, from next-generation surveys to probe the underlying flare SED (or its deviations) across the population of $\mathrm{M}$ dwarfs in the Galaxy. 
The authors thank C. MacLeod and J. Ruan for their insight in characterizing astrophysical variability, B. Tofflemire and J. Wisniewski for fruitful discussions regarding NIR flares, P. Kundurthy for his assistance in converting times to BJD, and the anonymous referee for helpful suggestions that greatly improved this manuscript. A.C.B. and J.R.A.D. acknowledge support from NASA ADP grant NNX09AC77G. E.J.H., S.L.H., and A.F.K. acknowledge support from NSF grant AST 08-07205.

This publication makes use of data products from the Two Micron All Sky Survey, which is a joint project of the University of Massachusetts and the Infrared Processing and Analysis Center, California Institute of Technology, funded by the National Aeronautics and Space Administration and the National Science Foundation.

This research has made use of the SIMBAD database, operated at CDS, Strasbourg, France.

Funding for the SDSS and SDSS-II has been provided by the Alfred P. Sloan Foundation, the Participating Institutions, the National Science Foundation, the U.S. Department of Energy, the National Aeronautics and Space Administration, the Japanese Monbukagakusho, the Max Planck Society, and the Higher Education Funding Council for England. The SDSS Web site is http://www.sdss.org/.

The SDSS is managed by the Astrophysical Research Consortium for the Participating Institutions. The Participating Institutions are the American Museum of Natural History, Astrophysical Institute Potsdam, University of Basel, University of Cambridge, Case Western Reserve University, University of Chicago, Drexel University, Fermilab, the Institute for Advanced Study, the Japan Participation Group, Johns Hopkins University, the Joint Institute for Nuclear Astrophysics, the Kavli Institute for Particle Astrophysics and Cosmology, the Korean Scientist Group, the Chinese Academy of Sciences (LAMOST), Los Alamos National Laboratory, the Max-PlanckInstitute for Astronomy (MPIA), the Max-Planck-Institute for Astrophysics (MPA), New Mexico State University, Ohio State University, University of Pittsburgh, University of Portsmouth, Princeton University, the United States Naval Observatory, and the University of Washington.

\section{REFERENCES}

Abazajian, K. N., Adelman-McCarthy, J. K., Agüeros, M. A., et al. 2009, ApJS, 182,543

Adelman-McCarthy, J. K., Agüeros, M. A., Allam, S. S., et al. 2008, ApJS, 175, 297

Allred, J. C., Hawley, S. L., Abbett, W. P., \& Carlsson, M. 2006, ApJ, 644, 484 Ankerst, M., Breunig, M. M., Kriegel, H.-P., \& Sander, J. 1999, in SIGMOD 1999, Proc. ACM SIGMOD Int. Conf. Manag. Data, ed. A. Delis, C. Faloutsos, \& S. Ghandeharizadeh (Philadelphia, PA: ACM Press), 49

Becker, A. C., Agol, E., Silvestri, N. M., et al. 2008, MNRAS, 386, 416

Bochanski, J. J., Hawley, S. L., Covey, K. R., et al. 2010, AJ, 139, 2679

Bochanski, J. J., Munn, J. A., Hawley, S. L., et al. 2007a, AJ, 134, 2418

Bochanski, J. J., West, A. A., Hawley, S. L., \& Covey, K. R. 2007b, AJ, 133, 531
Byrne, P. B. 1989, Sol. Phys., 121, 61

Cohen, M., Wheaton, W. A., \& Megeath, S. T. 2003, AJ, 126, 1090

Covey, K. R., Hawley, S. L., Bochanski, J. J., et al. 2008, AJ, 136, 1778

Covey, K. R., Ivezić, Ž., Schlegel, D., et al. 2007, AJ, 134, 2398

Cushing, M. C., Rayner, J. T., \& Vacca, W. D. 2005, ApJ, 623, 1115

Cutri, R. M., Skrutskie, M. F., Van Dyk, S., et al. 2006, Explanatory Supplement to the 2MASS All Sky Data Release and Extended Mission Products, http://www.ipac.caltech.edu/2mass/releases/allsky/doc/explsup.html

Fuhrmeister, B., Liefke, C., Schmitt, J. H. M. M., \& Reiners, A. 2008, A\&A, 487, 293

Fukugita, M., Ichikawa, T., Gunn, J. E., et al. 1996, AJ, 111, 1748

Hawley, S. L., Allred, J. C., Johns-Krull, C. M., et al. 2003, ApJ, 597, 535

Hawley, S. L., \& Fisher, G. H. 1992, ApJS, 78, 565

Hawley, S. L., \& Pettersen, B. R. 1991, ApJ, 378, 725

Hilton, E. J. 2011, PhD thesis, University of Washington

Hilton, E. J., Hawley, S. L., Kowalski, A. F., \& Holtzman, J. 2010a, arXiv:1012.0577

Hilton, E. J., West, A. A., Hawley, S. L., \& Kowalski, A. F. 2010b, AJ, 140, 1402

Ivezić, Ž., Smith, J. A., Miknaitis, G., et al. 2007, AJ, 134, 973

Ivezic, Z., et al. 2008, arXiv:0805.2366

Jenkins, J. S., Ramsey, L. W., Jones, H. R. A., et al. 2009, ApJ, 704, 975

Jenkins, L. F. (ed.) 1952, General Catalogue of Trigonometric Stellar Parallaxes (New Haven, CT: Yale Univ. Obs.)

Joy, A. H., \& Humason, M. L. 1949, PASP, 61, 133

Kowalski, A. F., Hawley, S. L., Hilton, E. J., et al. 2009, AJ, 138, 633

Kowalski, A. F., Hawley, S. L., Holtzman, J. A., Wisniewski, J. P., \& Hilton, E. J. 2010, ApJ, 714, L98

Kunkel, W. E. 1970, ApJ, 161, 503

Lacy, C. H., Moffett, T. J., \& Evans, D. S. 1976, ApJS, 30, 85

Loebman, S. R., Roskar, R., Debattista, V. P., et al. 2011, ApJ, 737, 8

Lovell, B. 1969, Nature, 222, 1126

Miller, C. J., Genovese, C., Nichol, R. C., et al. 2001, AJ, 122, 3492

Moffett, T. J. 1974, ApJS, 29, 1

Panagi, P. M., \& Andrews, A. D. 1995, MNRAS, 277, 423

Pickles, A. J. 1998, PASP, 110, 863

Plavchan, P., Gee, A. H., Stapelfeldt, K., \& Becker, A. 2008a, ApJ, 684 L37

Plavchan, P., Jura, M., Kirkpatrick, J. D., Cutri, R. M., \& Gallagher, S. C. 2008b, ApJS, 175, 191

Rau, A., Ofek, E. O., Kulkarni, S. R., et al. 2008, ApJ, 682, 1205

Rayner, J. T., Cushing, M. C., \& Vacca, W. D. 2009, ApJS, 185, 289

Reid, N., \& Hawley, S. L. (ed.) 2000, New Light on Dark Stars: Red Dwarfs, Low Mass Stars, Brown Dwarfs (Chichester: Praxis)

Robinson, R. D., Carpenter, K. G., \& Percival, J. W. 1999, ApJ, 516, 916

Robinson, R. D., Carpenter, K. G., Percival, J. W., \& Bookbinder, J. A. 1995, ApJ, 451, 795

Rodonò, M., \& Cutispoto, G. 1988, in Activity in Cool Star Envelopes, ed. O. Havnes, J. E. Solheim, B. R. Pettersen, \& J. H. M. M. Schmitt (Astrophysics and Space Science Library, Vol. 143; Dordrecht: Kluwer), 163

Schmidt, S. J. 2012, ApJ, 745, 14

Sesar, B., Ivezić, Ž., Lupton, R. H., et al. 2007, AJ, 134, 2236

Skrutskie, M. F., Cutri, R. M., Stiening, R., et al. 2006, AJ, 131, 1163

Stetson, P. B. 1996, PASP, 108, 851

Tofflemire, B. M., Wisniewski, J. P., Hilton, E. J., et al. 2012, AJ, 143, 12

van Altena, W. F., Lee, J. T., \& Hoffleit, E. D. (ed.) 1995, The General Catalogue of Trigonometric [Stellar] Parallaxes (New Haven, CT: Yale Univ. Obs.) van Leeuwen, F. 2007, A\&A, 474, 653

van Maanen, A. 1940, ApJ, 91, 503

Welch, D. L., \& Stetson, P. B. 1993, AJ, 105, 1813

West, A. A., Hawley, S. L., Bochanski, J. J., et al. 2008, AJ, 135, 785

West, A. A., Morgan, D. P., Bochanski, J. J., et al. 2011, AJ, 141, 97

Xu, Y., Cao, W., Liu, C., et al. 2006, ApJ, 641, 1210

York, D. G., Adelman, J., Anderson, J. E., Jr., et al. 2000, AJ, 120, 1579 